\title{
Phase Control of Nonadiabaticity-Induced Quantum Chaos in an Optical Lattice
}

\author{
Jiangbin Gong and Paul Brumer \\ Chemical Physics Theory Group, University of Toronto, Toronto, Canada M5S 3H6
}

(Received 27 September 2001; published 2 May 2002)

\begin{abstract}
The qualitative nature (i.e., integrable vs chaotic) of the translational dynamics of a three-level atom in an optical lattice is shown to be controllable by varying the relative laser phase of two standing-wave lasers. Control is explained in terms of the nonadiabatic transition between optical potentials and the corresponding regular-to-chaotic transition in mixed classical-quantum dynamics. The results are of interest to both areas of coherent control and quantum chaos.
\end{abstract}

DOI: $10.1103 /$ PhysRevLett.88.203001

Recent years have witnessed an increasing interest in the coherent control of atomic and molecular processes [1,2]. One central aspect of coherent control is phase control, in which different optical phases are introduced into coherent laser-matter interactions in order to manipulate quantum interference effects and thus to achieve target objectives. It has been shown that phase control approaches are widely applicable [1], even to some systems displaying quantum chaotic dynamics [3].

As we show in this Letter, optical lattices, of great recent interest [4], provide an important system in which to explore aspects of quantum chaos and coherent control. In particular, we describe the all-optical phase control of the translational motion of atoms in a system that allows chaotic vs integrable motion, depending on the phase shift between two standing-wave laser fields. The control mechanism is shown to originate in the nonadiabatic coupling between different optical potentials as well as in the regular-to-chaotic transition in a mixed classical-quantum description of the model system. The results are of broad interest to both coherent control and quantum chaos.
PACS numbers: $32.80 . \mathrm{Qk}, 05.45 . \mathrm{Gg}, 05.45 . \mathrm{Mt}$

Consider a $\Lambda$-type three-level atom moving along two copropagating standing-wave laser beams, with two lower degenerate levels $|1\rangle$ and $|3\rangle$, and one upper level $|2\rangle$. Two laser fields, with different polarizations $\sigma_{+}$and $\sigma_{-}$, couple $|1\rangle$ with $|2\rangle$ and $|2\rangle$ with $|3\rangle$, respectively. A closed three-level $\Lambda$ configuration of this type may be realized, for example, in ${ }^{4} \mathrm{He}$ using the $2{ }^{3} S_{1} \rightarrow 2{ }^{3} P_{1}$ transition [5]. The laser fields are of the same frequency, with large detuning $\Delta$ from $|2\rangle$. We use $x, p, M, \Omega_{1}\left(\Omega_{2}\right), k_{1}\left(=k_{2}\right)$ to represent the position, momentum, atomic mass, the two Rabi frequencies (assumed real), and the two wave vectors, respectively. The relative phase of the two standing waves is denoted by $\phi$. For generality we employ a set of natural units by scaling all the parameters, i.e., $x^{0}=\lambda$ for $x, p^{0}=\hbar / \lambda$ for $p, t^{0}=M \lambda^{2} / \hbar$ for the time variable $t$, $\Omega^{0}=2 / t^{0}$ for $\Delta, \Omega_{1}$, and $\Omega_{2}$. In terms of these units, the dynamical equations do not explicitly contain the atomic mass; the effective wave vector is given by $k=2 \pi$, and $[x, p]=i$ in the full quantum dynamics. In the rotating wave approximation and in the interaction picture, the Hamiltonian describing the translational motion along the laser beams is $H=p^{2} / 2+\left(V_{i j}\right)$, with the potential matrix $\left(V_{i j}\right)(i, j=1,2,3)$ given by

$$
\left(V_{i j}\right)=\left(\begin{array}{ccc}
2 \Delta & \Omega_{1} \sin (k x) & 0 \\
\Omega_{1} \sin (k x) & 0 & \Omega_{2} \sin (k x+\phi) \\
0 & \Omega_{2} \sin (k x+\phi) & 2 \Delta
\end{array}\right) .
$$

To demonstrate phase control of the dynamics, consider numerical results for the specific case where (i) $\Omega_{1}=$ $6.0 \times 10^{3}, \Omega_{2}=7.0 \times 10^{3}, \Delta=1.5 \times 10^{4}$, (ii) the atom is initially in an internal state $|1\rangle$, and (iii) the average momentum, average position, momentum variance, and position variance of the initial Gaussian wave packet are given by $\langle p\rangle=25.0,\langle x\rangle=0.0, \delta p=10 / \sqrt{2}$, and $\delta x=1.0 / 10 \sqrt{2}$, respectively. In ${ }^{4} \mathrm{He}$, this corresponds to $t^{0} \sim 77 \mu \mathrm{sec}$, the detuning $\sim 2 \pi \times 62 \mathrm{MHz}$ (about 38 times the linewidth of $|2\rangle)$, and the initial kinetic temperature $\sim 29 \mu \mathrm{K}$. Results for this case represent typical observations for a wide range of system parameters and initial conditions that we have examined [6].

The solid curves in Fig. 1, which contain the essential result of this Letter, show the time dependence of the quantum momentum expectation value $\langle p\rangle$ for time evolving wave packets for various $\phi$. The $\phi=0$ case (Fig. 1a) displays a perfectly regular recurrence pattern. By contrast, the $\phi=0.25 \pi$ case (Fig. 1b) is characteristic of a relaxation process, with significantly irregular oscillations of small amplitude. In addition, the associated power spectrum (not shown) is quite noisy, characteristic of chaos [7]. Further tuning $\phi$ leads to totally different dynamics: in the $\phi=0.5 \pi$ case (Fig. $1 \mathrm{c}$ ), $\langle p\rangle$ lies very close to its initial value and undergoes regular oscillations, but with a characteristic frequency that is much higher than that in the $\phi=$ 0 case (the dashed curves in Fig. 1 are discussed below). Clearly, the atom's translational motion undergoes significant qualitative changes with controlled changes in $\phi$.

To demonstrate that this is indeed an integrableto-chaotic transition, we consider the sensitivity of the 

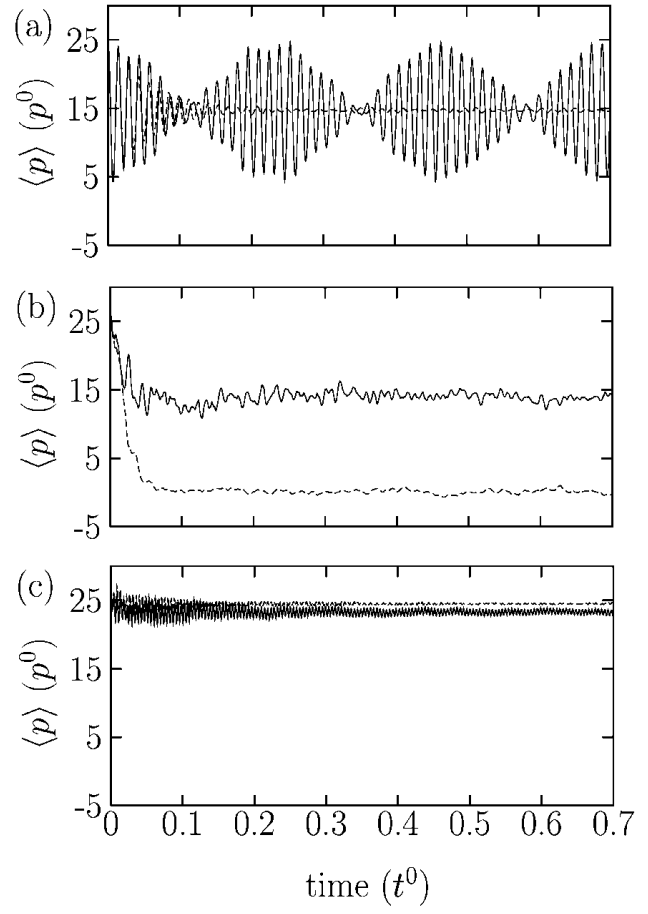

FIG. 1. Time dependence of $\langle p\rangle$ obtained from quantum wave packet dynamics calculations (solid lines) and the ensemble statistics in a mixed classical-quantum description (dashed lines). The initial internal state is $|1\rangle$, and the $\langle x\rangle$ and $\langle p\rangle$ of the initial Gaussian ensemble are 0.0 and 25.0, respectively. The initial variances in position and momentum are chosen to be $1.0 / 10 \sqrt{2}$ and $10.0 / \sqrt{2}$, respectively. The relative phase $\phi$ equals 0.0 , $0.25 \pi$, and $0.5 \pi$ in (a), (b), and (c), respectively.

dynamics to slight changes of $\phi$. Figure 2 shows the time dependence of the absolute value of the overlap $\chi$ of two time evolving wave functions emanating from the same initial Gaussian wave packet, with the relative phase of the two laser fields given by $\phi$ and $\phi+\pi / 400$. For $\phi=0$ or $\phi=0.5 \pi, \chi$ remains near unity throughout, indicating that the dynamics is insensitive to tiny changes

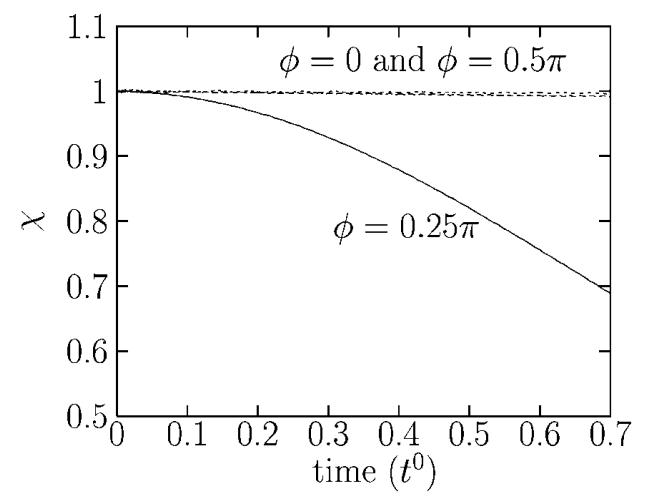

FIG. 2. The sensitivity of the quantum dynamics to slight changes of the relative phase parameter $\phi . \quad \chi$ is the absolute value of the overlap between the two time evolving wave functions emanating from the same initial state as in Fig. 1, with the relative phase of the two standing-wave laser fields given by $\phi$ and $\phi+\pi / 400$. of $\phi$. By contrast, for $\phi=0.25 \pi, \chi$ is already less than 0.70 at $t=0.7$. This interesting hypersensitivity [8] to perturbations in the relative phase parameter $\phi$ further suggests that the $\phi=0.25 \pi$ case is indicative of quantum chaos.

To further substantiate that this is a regular-to-chaotic transition we consider a mixed classical-quantum description of the dynamics, i.e., where the center-of-mass motion evolves classically on an average potential and where the internal motion is treated quantum mechanically $[9,10]$. From this perspective, the state of motion is described by a phase space point $(x, p)$ and an internal wave function $|\psi\rangle=\left(C_{1}, C_{2}, C_{3}\right)$, where $C_{1}, C_{2}$, and $C_{3}$ are the projections of the wave function onto the three internal levels. The dynamical equations are given by

$$
\begin{gathered}
\frac{d x}{d t}=p, \\
\frac{d p}{d t}=-\left\langle\psi\left|\frac{d V}{d x}\right| \psi\right\rangle \\
=-\Omega_{1} k\left(C_{2} C_{1}^{*}+C_{2}^{*} C_{1}\right) \cos (k x) \\
-\Omega_{2} k\left(C_{2} C_{3}^{*}+C_{2}^{*} C_{3}\right) \cos (k x+\phi),
\end{gathered}
$$

and

$$
i \frac{d C_{k}}{d t}=\sum_{j=1}^{3} V_{k j} C_{j}, \quad k=1,2,3 .
$$

One can readily solve these equations numerically to obtain $p(t)$ for the classical translational motion. The results, for the same system parameters and the same initial internal state as in the quantum calculations, but for a single trajectory initially at $(x, p)=(0.0,25.0)$, are shown in Fig. 3. In particular, for $\phi=0$ (Fig. 3a) the oscillation of momentum is perfectly regular, in agreement with the regular recurrence pattern in Fig. 1a. By contrast, for $\phi=0.25 \pi$ (Fig. 3b), the trajectory is highly irregular, with random alternations between fast smallamplitude and slow large-amplitude oscillations. In this case two initially nearby trajectories show exponential divergence in phase space with an associated Lyapunov exponent of $\sim 50 / t^{0}$. This supports the view that the irregular dynamics in Fig. 1b, and the sensitivity of the dynamics to slight changes of $\phi$ shown in Fig. 2, are indeed due to optical-phase-assisted quantum chaos. Further, in the $\phi=0.5 \pi$ case (Fig. 3c), the regular classical motion is restored, with a characteristic frequency identical to that in Fig. 1c. Hence optical-phase control is evident in this classical-quantum treatment as well.

An ensemble statistics in the classical treatment of translational motion provides further support. The dashed lines in Fig. 1 display the time dependence of the average momentum $\langle p\rangle$ for an ensemble of trajectories initially centered at $x=0$ and $p=25.0$, with the same initial variances as in the quantum calculations. Each individual trajectory in the ensemble is obtained by solving 

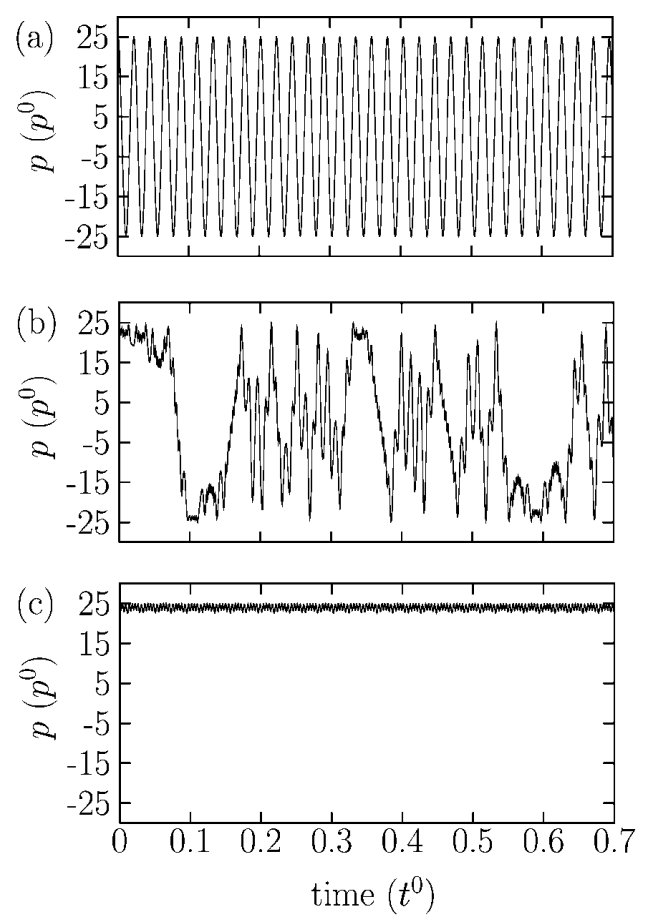

FIG. 3. Time dependence of momentum for classical trajectories obtained by solving Eqs. (2)-(4), with the initial position $x=0$ and the initial momentum $p=25.0$. The initial internal state is given by $|1\rangle$. $\phi$ equals $0.0,0.25 \pi$, and $0.5 \pi$ in (a), (b), and (c), respectively.

Eqs. (2)-(4). The quantum-classical correspondence for the regular dynamics at times $t<0.15$ in Figs. 1a and 1c is impressive. On the other hand, as seen in Fig. 1b, $\langle p\rangle$ for the classical ensemble quickly relaxes to zero, whereas $\langle p\rangle$ for the quantum ensemble remains far away from zero. This quantum-classical difference constitutes an excellent example of quantum suppression of classical chaos in an unbounded Hamiltonian system.

Insight into the origin of phase control can be obtained by considering the dynamics in an adiabatic representation. To do so we introduce an orthogonal transformation $\left(O_{i j}\right)(i, j=1,2,3)$ to diagonalize the potential matrix $\left(V_{i j}\right) .\left(O_{i j}\right)$ is given by

$$
\left(O_{i j}\right)=\left(\begin{array}{ccc}
\frac{\Omega_{1} \sin k x}{\sqrt{2\left(\eta^{2}-\Delta \eta\right)}} & \frac{\Omega_{2} \sin (k x+\phi)}{\xi} & \frac{\Omega_{1} \sin (k x)}{\sqrt{2\left(\eta^{2}+\Delta \eta\right)}} \\
\frac{\eta-\Delta}{\sqrt{2\left(\eta^{2}-\Delta \eta\right)}} & 0 & \frac{-\eta-\Delta}{\sqrt{2\left(\eta^{2}+\Delta \eta\right)}} \\
\frac{\Omega_{2} \sin (k x+\phi)}{\sqrt{2\left(\eta^{2}-\Delta \eta\right)}} & \frac{-\Omega_{1} \sin (k x)}{\xi} & \frac{\Omega_{2} \sin (k x+\phi)}{\sqrt{2\left(\eta^{2}+\Delta \eta\right)}}
\end{array}\right),
$$

where $\xi(x, \phi) \equiv \sqrt{\Omega_{1}^{2} \sin ^{2}(k x)+\Omega_{2}^{2} \sin ^{2}(k x+\phi)}$ and $\eta(x, \phi) \equiv \sqrt{\xi^{2}(x, \phi)+\Delta^{2}}$. Corresponding to the three eigenvectors $\left(O_{1 j}, O_{2 j}, O_{3 j}\right)(j=1,2,3)$, are three eigenpotentials $V_{i}(x, \phi)$ given by $V_{1}(x, \phi)=\Delta+\eta(x, \phi)$, the constant potential $V_{2}=2 \Delta$, and $V_{3}(x, \phi)=\Delta-$ $\eta(x, \phi)$. For the special case of $\phi=0, V_{1}$ and $V_{2}$ are degenerate at $k x=n \pi$, and the eigenvector $\left(O_{12}, O_{22}, O_{32}\right)$ is $x$ independent; for the general cases of $\phi \neq 0$, $V_{1}(x, \phi)>V_{2}>V_{3}(x, \phi)$, i.e., the three potential curves do not cross each other. Of particular interest is the constant potential $V_{2}$, associated with the eigenvector $\left(O_{12}, O_{22}, O_{32}\right)$. Since

$$
\Omega_{1} \sin (k x) O_{12}+\Omega_{2} \sin (k x+\phi) O_{32}=0,
$$

$V_{2}$ results from the complete quantum destructive interference between the two standing-wave laser fields. As such, $V_{2}$ is an extension of the "dark optical lattice" in the presence of two counterpropagating plane-wave laser beams $[5,11,12]$.

Consider now transforming Eqs. (3) and (4) to the eigenpotential (adiabatic) representation. Specifically, consider the dynamics in terms of $\tilde{C}_{i}, i=1,2,3$, where $\tilde{C}_{i}=$ $\sum_{k=1}^{3} O_{k i} C_{k}$. Using $\sum_{k=1}^{3} O_{k i} O_{k j}=\delta_{i j}$ and $\sum_{k=1}^{3} O_{k i} \times$ $d O_{k j} / d x=-\sum_{k=1}^{3} O_{k j} d O_{k i} / d x$, Eqs. (3) and (4) can be transformed to

$$
\frac{d p}{d t}=-\left|\tilde{C}_{1}\right|^{2} \frac{d V_{1}(x, \phi)}{d x}-\left|\tilde{C}_{3}\right|^{2} \frac{d V_{3}(x, \phi)}{d x},
$$

and

$$
i\left(\begin{array}{c}
\frac{d \tilde{C}_{1}}{d t} \\
\frac{d \tilde{C}_{2}}{d t} \\
\frac{d \tilde{C}_{3}}{d t}
\end{array}\right)=\left(\begin{array}{ccc}
V_{1}(x, \phi) & i t_{12} & -i t_{13} \\
-i t_{12} & V_{2} & -i t_{23} \\
i t_{13} & i t_{23} & V_{3}(x, \phi)
\end{array}\right)\left(\begin{array}{l}
\tilde{C}_{1} \\
\tilde{C}_{2} \\
\tilde{C}_{3}
\end{array}\right),
$$

where the diagonal terms are the three adiabatic potentials given above, and the potential coupling terms are given by $t_{12}(x, \phi)=p k \Omega_{1} \Omega_{2} \sin (\phi) /\left[2 \xi^{2}\left(\eta^{2}-\Delta \eta\right)\right]^{1 / 2}, t_{13}(x$, $\phi)=p k \Delta\left[\Omega_{1}^{2} \sin (2 k x)+\Omega_{2}^{2} \sin (2 k x+2 \phi)\right] / 4 \eta^{2} \xi$, and $t_{23}(x, \phi)=p k \Omega_{1} \Omega_{2} \sin (\phi) /\left[2 \xi^{2}\left(\eta^{2}+\Delta \eta\right)\right]^{1 / 2} \quad[13]$. Note that $t_{i j}(i \neq j=1,2,3)$ is proportional to the momentum $p$. Thus, the coupling between optical potentials is due to the nonadiabatic effects associated with translational motion. Further, for the computational example discussed above (and many other cases in which $\Delta>0$ ), one has $\min \left[V_{1}(x, \phi)-V_{2}\right] \ll \min \left[V_{2}-V_{3}(x, \phi)\right]$ and $t_{12}(x, \phi) \gg t_{23}(x, \phi)$, suggesting that $V_{3}(x, \phi)$ is effectively decoupled from $V_{1}(x, \phi)$ and $V_{2}$. Note also that, at the initial location $x=0$, the initial internal state $|1\rangle$ is a superposition state of the two eigenvectors associated with $V_{1}$ and $V_{2}$ for $\phi=0$, and reduces to the eigenvector associated with $V_{2}$ for $\phi \neq 0$.

The key role of the relative laser phase $\phi$ becomes clear as one compares the magnitude of the nonadiabatic coupling term $t_{12}(x, \phi)$ with that of $\left(V_{1}-V_{2}\right)$. For cases 1a and $3 \mathrm{a}, \phi=0$ and $t_{12}(x, \phi=0)=0$, so the dynamics is adiabatic. Thus, in the case of Fig. 1a, the quantum ensemble divides into two subensembles: one is trapped in one well of $V_{1}$ around $x=0$ and undergoes periodic oscillations, and the other experiences the trivial motion on the constant potential $V_{2}$. For cases $1 b$ and $3 b$, $\phi=0.25 \pi$. Here the smallest gap between $V_{1}$ and $V_{2}$ 
is given by $g(\phi)=\sqrt{A^{2}(\phi)+\Delta^{2}}-\Delta$, where $A^{2}(\phi)=$ $\left[\Omega_{1}^{2}+\Omega_{2}^{2}-\sqrt{\Omega_{1}^{4}+\Omega_{2}^{4}+2 \Omega_{1}^{2} \Omega_{2}^{2} \cos (2 \phi)}\right] / 2$. The corresponding ratio of the potential coupling term $t_{12}$ to $g(\phi)$ is given by

$$
T(\phi)=\frac{p k \Omega_{1} \Omega_{2} \sin (\phi)}{\sqrt{2}\left[A^{2}(\phi)+\Delta^{2}\right]^{1 / 4} A(\phi) g^{3 / 2}(\phi)} .
$$

Since $T(\phi=0.25 \pi) \approx 1.0$, the magnitude of the nonadiabatic coupling is comparable to that of $\left(V_{1}-V_{2}\right)$, resulting in strong nonadiabatic effects. Thus, the chaotic motion seen in Fig. $3 \mathrm{~b}$ is induced by the significant nonadiabatic coupling between the two simple onedimensional potentials $V_{1}$ and $V_{2}$. Finally, for cases $1 \mathrm{c}$ and $3 \mathrm{c}, \phi=0.5 \pi$. Here $T(\phi=0.5 \pi)=\min [T(\phi)] \approx$ 0.16 , i.e., the nonadiabatic coupling is appreciably weaker than in the case of $\phi=0.25 \pi$. As such, the translational motion, initially launched on the adiabatic potential $V_{2}$, would essentially remain on $V_{2}$, with small perturbations from the insignificant Rabi population oscillation between $V_{1}$ and $V_{2}$. To further confirm this picture, one finds that the characteristic frequency of the regular dynamics in Figs. $1 \mathrm{c}$ and $3 \mathrm{c}$ is $\sim 1425$, a value consistent with the Rabi frequencies given by $\sqrt{\left(V_{1}-V_{2}\right)^{2}+4 t_{12}^{2}}$ [see Eq. (8)].

A number of additional remarks are in order. First, the two-standing-wave configuration is essential in this system. That is, if either or both of the two standing-wave fields are replaced by a traveling wave, the nonadiabatic coupling or the spacing between $V_{1}$ and $V_{2}$ is no longer a sensitive function of $\phi$, and there is no significant phase control. On the other hand, a three-level atom in two standing-wave laser fields of different but commensurate frequencies also shows dynamics that is controllable by changing $\phi$ [14]. Second, in contrast to some recent studies on an optical-magneto lattice $[15,16]$, the coupling between different optical potentials discussed above is not due to additional magnetic fields, but directly due to nonadiabaticity. Further, unlike the work in Ref. [16], here we have observed clear signatures of quantum chaos in the quantum dynamics. Thus, this model is the first alloptics realization of nonadiabaticity-induced quantum chaos, a phenomenon first discovered in molecular systems [17]. Third, in this paper we have neglected decoherence effects (e.g., due to the spontaneous emission from the excited state $|2\rangle)$. It would be interesting to explore how decoherence affects phase control and quantum-classical correspondence in this system.

In conclusion, we have demonstrated optical-phase control of nonadiabaticity-induced quantum chaos in a $\Lambda$-type three-level system in a two-standing-wave optical lattice. The results shown in Fig. 1 are only samples of the observable, controllable, behavior. Further, the functional depen- dence on $\phi$ has been exposed analytically, which can serve to guide experimental studies of the $\phi$-dependent regularto-chaotic transition. Recent experimental progress in atom optics and quantum chaos $[4,5,12,15,18]$ suggests that the results should be experimentally achievable with existent technology.

This work was supported by the U.S. Office of Naval Research and the Natural Sciences and Engineering Research Council of Canada.

[1] M. Shapiro and P. Brumer, Adv. At. Mol. Opt. Phys. 42, 287 (2000).

[2] S. A. Rice and M. Zhao, Optical Control of Molecular Dynamics (John Wiley, New York, 2000).

[3] J. Gong and P. Brumer, Phys. Rev. Lett. 86, 1741 (2001); J. Chem. Phys. 115, 3590 (2001); D. Abrashkevich, M. Shapiro, P. Brumer, J. Chem. Phys. (to be published).

[4] F. L. Moore et al. Phys. Rev. Lett. 75, 4598 (1995); B. G. Klappauf et al. Phys. Rev. Lett. 81, 1203 (1998); H. Ammann et al. Phys. Rev. Lett. 80, 4111 (1998); J. Ringot et al., Phys. Rev. Lett. 85, 2741 (2000); M. B. dArcy et al. Phys. Rev. Lett. 87, 074102 (2001).

[5] A. Aspect et al. Phys. Rev. Lett. 61, 826 (1988).

[6] The system parameters and the initial conditions should be chosen to ensure that the population on level $|2\rangle$ is small $(<2 \%$ in the present case) so that spontaneous emission effects will be insignificant. It is also desirable to ensure negligible tunneling effects between different wells of $V_{1}$ so as to achieve good quantum-classical correspondence.

[7] R. S. Dumont and P. Brumer, J. Chem. Phys. 88, 1481 (1988).

[8] R. Schack and C.M. Caves, Phys. Rev. Lett. 71, 525 (1993); A. Peres, Phys. Rev. A 30, 1610 (1984).

[9] J. C. Tully and R. K. Preston, J. Chem. Phys. 55, 562 (1971).

[10] R. Blümel and B. Esser, Phys. Rev. Lett. 72, 3658 (1994).

[11] R. Dum and M. Olshanii, Phys. Rev. Lett. 76, 1788 (1996).

[12] S. K. Dutta, B. K. Teo, and G. Raithel, Phys. Rev. Lett. 83, 1934 (1999).

[13] The mixed classical-quantum treatment automatically neglects the optical gauge potential [11,12], which is not of much interest as far as the nonadiabatic coupling is concerned.

[14] J. Gong and P. Brumer (to be published).

[15] D. L. Haycock et al. Phys. Rev. Lett. 85, 3365 (2000).

[16] S. Ghose, P.M. Alsing, and I. H. Deutsch, quan-ph/ 0102085.

[17] E. J. Heller, J. Chem. Phys. 92, 1718 (1990); H. Schanz and B. Esser, Phys. Rev. A 55, 3375 (1997); H. Fujisaki and K. Takatsuka, Phys. Rev. E 63, 066221 (2001).

[18] D. A. Steck, W. H. Oskay, and M. G. Raizen, Science 293, 274 (2001); W. K. Hensinger et al., Nature (London) 412, 52 (2001). 